\title{
ANÁLISIS DE LA RELACIÓN ENTRE APOYO SOCIAL Y LESIONES EN FUTBOLISTAS FEDERADOS
}

\section{ANALYSIS OF THE RELATIONSHIP BETWEEN SOCIAL SUPPORT AND INJURIES IN FEDERATED FOOTBALL PLAYERS}

\author{
VERÓNICA GÓMEZ-ESPEJO ${ }^{1}$, IRMA ÁlVAREZ ${ }^{1}$, LUCÍA ABENZA² Y \\ AURELIO OLMEDILLA ${ }^{1}$
}

Cómo referenciar este artículo/How to reference this article:

Gómez-Espejo, V., Álvarez, I., Abenza, L. y Olmedilla, A. (2017). Análisis de la relación entre apoyo social y lesiones en futbolistas federados [Analysis of the Relationship between Social Support and Injuries in Federated Football Players]. Acción Psicológica, 14(1), 57-64. http://dx.doi.org/10.5944/ap.14.1.19261

\section{Resumen}

El objetivo de este estudio fue determinar si había diferencias entre un grupo de futbolistas que han estado lesionados la temporada anterior y uno de futbolistas que no han estado lesionados en los niveles de apoyo social percibido por parte de los jugadores. La muestra estuvo formada por 219 jugadores sénior de fútbol (80.1\%) y fútbol sala (19.9\%) federados (de categorías primera, segunda, autonómica, preferente y categorías inferiores) a nivel regional. De ellos, 138 futbolistas formaron el grupo de aquellos que no sufrieron ninguna lesión deportiva durante la temporada anterior y 81 que sufrieron una o más lesiones. Las variables de estudio fueron el apoyo social percibido y las lesiones deportivas, evaluadas por la Escala Multidimensional de Apoyo Social Percibido de Landeta y Calvete (2002) y un instrumento ad hoc, respectivamente. Los resultados muestran que el grupo de futbolistas no lesionados, respecto al grupo de futbolistas lesionados, poseía niveles más altos de apoyo social en los tres ámbitos de éste (apoyo de los amigos $M=24.05$ frente a $M=23.77$;

Agradecimientos: Este trabajo se ha realizado gracias a la ayuda del Proyecto de la Federación de Fútbol de la Región de Murcia y la Universidad de Murcia, Football Project FFRM+UMU (04 0092 321B 64502 14704).

Correspondencia: Verónica Gómez-Espejo. Programa Internacional Doctorado Psicología. Universidad de Murcia. Campus de Espinardo. 30100 Espinardo, Murcia. Email: veronica.gomez2@um.es

ORCID: Aurelio Olmedilla (http://orcid.org/0000-0002-2389-0515).

${ }^{1}$ Universidad de Murcia, España.

${ }^{2}$ Universidad Católica San Antonio de Murcia, España. 
apoyo de la familia $M=24.71$ frente a $M=24.19$; y apoyo de otras personas relevantes $M=24.74$ frente a $M=23.71$ ), aunque dichas diferencias no fueron estadísticamente significativas $(p=.708 ; \quad p=.443$; $p=.143$ ). No se puede afirmar que la provisión de apoyo social a los jugadores de fútbol sirva para prevenir las lesiones deportivas, aunque sí existen diferencias entre el grupo de lesionados y el grupo de no lesionados, pero éstas no han sido significativas.

Palabras clave: lesiones deportivas; apoyo social; futbolistas.

\begin{abstract}
The aim of this study was to determine if there were differences between a group of players who have been injured the previous season and one of players who have not been injured in the levels of social support perceived by the players. The sample consisted of 219 senior football players $(80.1 \%)$ and federated football $(19.9 \%)$ at the regional level. Of these, 138 football players formed the group of those who did not suffer any sport injury during the previous season and 81 who suffered one or more injuries. The study variables were perceived social support and sports injuries, assessed by the Multidimensional Scale of Social Perceived Support (Landeta y Calvete, 2002) and an ad hoc instrument, respectively. The results show that the group of players non-injured players, compared to the group of injured players, had higher levels of social support in three areas of this (friends support $M=24.05$ vs. $M=23.77$; family support $M=24.71$ vs. $M=24.19$; and support from other relevant people $M=24.74$ vs. $M=23.71$ ), although these differences were not statistically significant $(p=.708$; $p=.443 ; p=.143$ ). We cannot say that the provision of social support for soccer players serve to prevent sports injuries, although there are differences between the group of injured and uninjured group, but these have not been significant.
\end{abstract}

Keywords: sport injuries; social support; football players.

\section{Introducción}

La lesión es un hecho inherente a la práctica deportiva, y como tal existen numerosos estudios epidemiológicos que muestran la gran prevalencia de lesiones en el deporte (Azuara, Flores y Alcolea, 2014; Hurtubise, Beech y Macpherson, 2015; Padegimas, Stepan, Stoker, Polites y Brophy, 2016; Pujals, Rubio, Márquez, Sánchez-Iglesias y Ruiz, 2016; Sheu, Chen y Hedegaard, 2016). Por otro lado, la mayoría de deportistas han sufrido alguna lesión durante su carrera deportiva suponiendo, a veces, lesiones graves que ocasionan un gran impacto en su salud y bienestar y en su desarrollo deportivo, además del elevado coste económico que estas suponen (Almeida, Olmedilla, Rubio y Palou, 2014; Udry y Andersen, 2008).

Las causas de las lesiones son múltiples y se encuentra agrupadas en intrínsecas y extrínsecas, por esto, la mayoría de autores proponen una perspectiva multidimensional en el análisis de los factores de predis-posición, pero no de causación (Meeuwisse, Tyreman, Hagel y Emery, 2007). Dentro de las dimensiones probables de factores de riesgo asociados se encuentran los factores psicosociales. Éstos se han convertido en un área de gran interés en la investigación de las dos últimas décadas, llegando a ocupar un rol significativo tanto para explicar la ocurrencia de lesiones, como para comprender mejor el proceso de recuperación de las mismas (Almeida et al., 2014; Fernandes et al. 2014; Johnson e Ivarsson, 2011; Maddison y Prapavessis, 2005; Petrie, Deiters y Harmison, 2014).

En relación a esta cuestión, como se verá más adelante, el modelo de estrés y lesión de Andersen y Williams (1988) fue el que marcó gran parte de la investigación a lo largo de los años y que todavía hoy sigue siendo un referente en el área. Según estos autores, la respuesta de estrés ante las demandas competitivas y otras facetas de la vida del deportista es un factor clave en la patogénesis de la lesión. La respuesta de estrés del deportista produce un incremento de la tensión muscular, que, a su vez, puede perjudicar la coordinación motora y reducir la flexibilidad. Además, la respuesta de estrés también puede inducir déficits atencionales; en concreto, la reducción del campo visual, con pérdida de informa- 
ción periférica relevante, o la distracción. La respuesta al estrés de los deportistas puede ser moderada por tres factores principales: la personalidad, la historia de factores de estrés y los recursos de afrontamiento. Estas variables psicosociales pueden atenuar o agravar la capacidad de respuesta de estrés que afecta a la vulnerabilidad del deportista a la lesión (Petrie y Perna, 2004).

En relación a esto y continuando con las variables psicosociales, hay suficiente evidencia empírica que constata que los factores psicosociales pueden desempeñar un papel como antecedentes de la lesión deportiva (Johnson, 2007), pero también pueden verse afectadas como consecuente, tal y como indican Wiese-Bjornstal, Smith, Shaffer y Morrey (1998) en su modelo integrado de la respuesta psicológica a la lesión y al proceso de rehabilitación, y afectar directamente a las conductas de adherencia a los programas de rehabilitación. Se podría considerar una relación de estrés-lesión-estrés, o factor psicológico-lesión-factor psicológico, en la que el deportista entra en un bucle de difícil solución, en la línea de lo propuesto por Olmedilla y García-Mas (2009) en su modelo global psicológico de las lesiones deportivas, y lo encontrado en el estudio de Olmedilla, Ortega y Gómez (2014).

En relación al tema que nos compete, el apoyo social es una de las variables de los recursos de afrontamiento de los deportistas que atendiendo al modelo de Andersen y Williams (1988) modera la relación estrés-lesión (Yang, Peek-Asa, Lowe, Heiden y Foster, 2010; Yang et al., 2014). Algunos estudios proporcionaron resultados que sostenían que la familia fue la fuente más importante de apoyo social (Petrie et al., 2014; Covassin et al., 2014). Así, en el trabajo de Petrie et al. (2014) se encontró que el apoyo social familiar junto con el bajo nivel de resistencia mental y el estrés producido por eventos positivos de la vida diaria, se relacionaba significativamente con el aumento de lesiones; es decir, que los deportistas que tienen bajos niveles de resistencia mental y de apoyo social familiar se lesionan con mayor frecuencia cuando experimentan altos niveles de estrés positivo.

Sin embargo, dichos estudios científicos han mostrado resultados poco concluyentes al respecto. $\mathrm{Si}$ bien algunos indican que una mayor red de apoyo social del deportista, minimiza el riesgo que éste tiene de sufrir lesión deportiva (Hardy, Richman y Rosenfeld, 1991; Johnson e Ivarsson, 2011; Petrie et al., 2014), otros estudios no establecieron ninguna relación entre estos factores (Malinauskas, 2010; Rees, Mitchel, Evans y Hardy, 2010). Quizá, la inconsistencia de estos resultados probablemente sea debida a que el papel mediador del apoyo social es más complejo que el propuesto inicialmente en el modelo de Andersen y Williams (1988).

En conclusión, el estudio del apoyo social y su influencia en las lesiones deportivas sigue siendo un ámbito de importancia para la investigación en el cual no se obtienen resultados concluyentes, requiriéndose profundizar sobre la temática. El objetivo de este trabajo es determinar si existen diferencias en los niveles percibidos de apoyo social entre un grupo de futbolistas que han estado lesionados y un grupo de futbolistas que no han estado lesionados la temporada anterior.

Para ello, se partió de la hipótesis de que existirían diferencias en los niveles de apoyo social percibido de aquellos deportistas que habían sufrido una lesión la temporada anterior respecto a los que no la sufrieron, concretamente, aquellos jugadores de futbol y futbol sala que no habían sufrido una lesión durante la temporada anterior percibirían mayores niveles de apoyo social.

\section{Método}

\section{Participantes}

La muestra estuvo formada por 219 futbolistas españoles federados, con edades comprendidas entre $14 \mathrm{y}$ 27 años $(M=22.5 \pm 4.6$, hombres; $M=16.5 \pm 2.2$, mujeres). Pertenecían a equipos federados de fútbol y fútbol sala de una provincia del sureste español (Región de Murcia). De ellos un $80.1 \%$ practicaba fútbol $(n=175)$ y un $19.9 \%(\mathrm{n}=44)$ practicaba fútbol sala. En lo referente a la categoría deportiva, un $9.1 \%(n=20)$ eran de primera categoría, un $30.1 \%(n=66)$ eran de segunda categoría, un $41.1 \% \quad(n=90)$ eran de categoría autonómica, un $6.8 \% \quad(\mathrm{n}=15)$ eran de categoría 
preferente y un $12.8 \%(\mathrm{n}=28)$ eran de otras categorías inferiores. Del total de la muestra un $63 \%(\mathrm{n}=138)$ de los jugadores no sufrieron ninguna lesión la temporada anterior y un $37 \%(\mathrm{n}=81)$ sufrieron una o más lesiones. La media de días de entreno era de $2.63( \pm .56)$ días, el tiempo medio de los entrenos era de $102.5( \pm 15.09)$ horas y el tiempo medio semanal empleado en los entrenos era de $267.65( \pm 68.16)$ horas.

\section{Instrumentos de evaluación}

Para evaluar la lesión deportiva se utilizó un instrumento ad hoc, cuyo protocolo está basado en el registro de lesiones y definición de lesión de Fuller, Ekstrand y Junge (2006) y de Junge et al. (2009), utilizado por el Comité Olímpico Internacional (COI) y por la Federation International of Football Association (FIFA), y en otros trabajos de investigación (GarcíaMas, Rubio, Fuster-Parra, Núñez y Pujals, 2014). En él se recogen el número de lesiones padecidas la temporada pasada y para cada una de ellas, la fecha en la que sucedió y el tiempo empleado en volver a practicar el deporte, el tipo de lesión (muscular, fractura/fisura, tendinitis, contusión, esguince $\mathrm{y} / \mathrm{u}$ otras), la situación (entrenamiento, partido, otros), una descripción detallada de la lesión, la causa (uno mismo, adversario en partido, compañero, material/equipamiento, otros), la gravedad (leve, moderada, grave, muy grave) y la afectación a la práctica deportiva (numero de entrenamiento perdidos, numero de partidos perdidos).

Para evaluar el apoyo social se utilizó la Escala Multidimensional de Apoyo Social Percibido de Landeta y Calvete (2002), adaptación española de la Perceived Social Support Multidimmensional Scale de Zimet, Dahlem, Zimet y Farley (1988) cuya escala global obtuvo un alfa de Cronbach de .85. Este instrumento consta de 12 ítems en forma de afirmación con siete opciones de respuesta tipo Likert $(1=$ Totalmente en desacuerdo; $7=$ Totalmente de acuerdo) que evalúa 3 sub-escalas (Familia, Amigos y Personas).

\section{Procedimiento}

Se realizó un tipo de muestreo incidental y se solicitó a la Federación de Fútbol la relación de clubes federados con equipos senior. Se contactó con los directores deportivos y/o los entrenadores de los clubes seleccionados para concertar una cita donde informar de la investigación a jugadores y entrenadores. Una vez obtenido el permiso y visto bueno para el estudio, se acordó un día para informar a los jugadores, explicándoles el objetivo de la investigación y las posibles dudas sobre la misma; se les informó del carácter voluntario de su participación y de la necesidad de firmar un consentimiento informado en caso de su participación. Para los menores de edad se les facilitó un consentimiento informado que deberían de firmar sus padres o tutores. Por último, se distribuyeron los cuestionarios informando del procedimiento a seguir para su cumplimentación. Se necesitaron aproximadamente 25 minutos. Todas las reuniones se llevaron a cabo, tras la finalización del entrenamiento, en los vestuarios (campo de fútbol, cancha fútbol sala) con todo el equipo, y la labor de explicación y desarrollo de la cumplimentación de los cuestionarios fue llevada a cabo por psicólogos expertos en deporte.

\section{Diseño y análisis de datos}

Se usó un diseño transversal, descriptivocorrelacional. Se utilizó el paquete estadístico SPSS 18.0. Para el análisis descriptivo de la muestra, se obtuvieron las medias, desviaciones típicas y frecuencias absolutas y relativas. Para la comparativa entre los grupos de lesionados y no lesionados la temporada anterior, se realizó una comparación de medias a través del estadistico $t$ de Student para muestras independientes con $p<.05$, previa comprobación de igualdad de varianzas con la prueba de Levene.

\section{Resultados}

Los datos indican diferencias entre deportistas que sufrieron una lesión la temporada anterior y los que no la sufrieron, aunque no llegan a la significación estadística. En concreto, los que no sufrieron ninguna lesión la tem- 
Tabla 1

Estadísticos descriptivos de grupo respecto a tres redes de apoyo social

\begin{tabular}{llrrr}
\hline Variable & Grupo & $\mathrm{N}$ & $\mathrm{M}$ & $\mathrm{SD}$ \\
\hline \multirow{2}{*}{ Apoyo social: amigos } & No lesionado & 136 & 24.05 & 4.97 \\
& Lesionado & 78 & 23.77 & 5.82 \\
Apoyo social: familia & No lesionado & 135 & 24.71 & 4.23 \\
\multirow{2}{*}{ Apoyo social: otros } & Lesionado & 79 & 24.19 & 5.62 \\
& No lesionado & 134 & 24.74 & 4.41 \\
\hline
\end{tabular}

porada anterior obtienen mayores puntuaciones en Apoyo social en las sub-escalas familia, amigos y otros frente a los que no sufrieron ninguna lesión la temporada pasada. Los resultados se presentan en la Tabla 1.

Con la $t$ de Student para muestras independientes, tanto asumiendo como no varianzas iguales, no se observaron diferencias estadísticamente significativas en el grupo de lesionados y no lesionados en ninguna de las redes de apoyo social analizadas (asumidas varianzas iguales: amigos $p=.708$; familia $p=.443$; otros $p=.143$ ), tal y como se puede ver en la Tabla 2 .

\section{Discusión}

El objetivo del estudio fue comprobar si la percepción de apoyo social, en cualquiera de sus ámbitos (familiar, amigos, otros) era diferente en futbolistas que habían sufrido una lesión y los que no la habían sufrido, y si estas diferencias eran estadísticamente significativas. Para ello, se partió de la hipótesis de que el grupo de futbolistas que habían sufrido una lesión la temporada anterior percibían un nivel de apoyo social menor que el de futbolistas que no habían sufrido una lesión.

Los resultados del estudio muestran que el grupo de los futbolistas que no sufrieron ninguna lesión la tempo- rada anterior obtuvieron puntuaciones mayores en los tres ámbitos del apoyo social, si bien las diferencias con el grupo de futbolistas que si sufrieron una lesión no fueron estadísticamente significativas. En esta línea de resultados aparecen estudios en los que, efectivamente, los deportistas con mayor nivel de apoyo social tuvieron menor número de lesiones que aquellos con un menor nivel de apoyo social (Hardy et al., 1991; Olmedilla, García-Montalvo y Martínez-Sánchez, 2006; Yang et al., 2014).

De esta manera el apoyo social puede considerarse un factor a tener en cuenta tanto en la prevención como en la rehabilitación de lesiones (Bianco, 2001; Robbins y Rosenfeld, 2001). Parece, en definitiva, que la tendencia que muestran los resultados de este trabajo está en la línea de que la provisión de apoyo social puede ser importante en la prevención de lesiones deportivas, y dado que las diferencias encontradas no han sido significativas sigue manteniendo la controversia al respecto. En este sentido, el estudio de la relación entre el apoyo social y las lesiones deportivas sigue siendo un ámbito importante de investigación en el que es necesario profundizar algo más.

Quizá algunas limitaciones de la presente investigación impidan observar mayor contundencia estadística. La tendencia muestra que es posible que se necesite de un mayor número muestral, y de una mayor

Tabla 2

Prueba T de Student para la igualdad de medias en muestras independientes

\begin{tabular}{|c|c|c|c|c|}
\hline Variable & Grupo & $t$ & $\mathrm{gl}$ & Sig. (bilateral) \\
\hline \multirow{2}{*}{ Apoyo social: amigos } & Asumidas varianzas iguales & .375 & 212 & .708 \\
\hline & No asumidas varianzas iguales & .359 & 140.85 & .720 \\
\hline \multirow{2}{*}{ Apoyo social: familia } & Asumidas varianzas iguales & .768 & 212 & .443 \\
\hline & No asumidas varianzas iguales & .714 & 129.92 & .477 \\
\hline \multirow{2}{*}{ Apoyo social: otros } & Asumidas varianzas iguales & 1.471 & 212 & .143 \\
\hline & No asumidas varianzas iguales & 1.379 & 134.99 & .170 \\
\hline
\end{tabular}


homogeneización, sobre todo respecto al género de los participantes y al deporte practicado. Tal y como indican Johnson, Tranaeus e Ivarsson (2014) si bien se han realizado progresos en la investigación de los antecedentes psicológicos para la prevención e intervención de las lesiones deportivas en los últimos 10-15 años, todavía quedan por desarrollar algunos aspectos metodológicos, de los que cabría destacar la incorporación de diseños experimentales, diseños de medidas repetidas y el uso de estadísticas que puedan probar interacciones complejas y diferencias intra-individuales.

Por último, indicar que este tipo de avances en la investigación permitirá identificar factores que predisponen a una mayor vulnerabilidad a la lesión y, por ende, identificar a deportistas con alto riesgo de lesión con el fin de diseñar programas de prevención e intervención de lesiones deportivas (Almeida et al., 2014; Johnson et al., 2014; Olmedilla, 2005; Olmedilla, Rubio, Ortega y García-Mas, 2017).

\section{Referencias}

Almeida, P. L., Olmedilla, A, Rubio, V. J. y Palou, P. (2014). Psychology in the Realm of Sport Injury. Revista de Psicología del Deporte/Journal of Sport Psychology, 23(2), 395-400.

Andersen, M. B. y Williams, J. M. (1988). A Model of Stress and Athletic Injury: Prediction and Prevention. Journal of Sport and Exercise Psychology, 10(3), 294-306. http://dx.doi.org/10.1123/jsep.10.3.294

Azuara, D. R., Flores, D. P. y Alcolea, M. R. (2014). Epidemiology of Sports Injuries in European Union Countries. Revista Internacional de Medicina y Ciencias de la Actividad Física y del Deporte, 14(55), 479-494.

Bianco, T. (2001). Social Support and Recovery from Sport Injury: Elite Skiers Share their Experiences. Research Quarterly for Exercise and
Sport, 72(4),

376-388.

http://dx.doi.org/10.1080/02701367.2001.10608974

Covassin, T., Crutcher, B., Bleecker, A., Heiden, E., Dailey, A. y Yang, J. (2014). Postinjury Anxiety and Social Support Among Collegiate Athletes: A Comparison Between Orthopaedic Injuries and Concussions. Journal of Athletic Training, 49(4), 462-468. http://dx.doi.org/10.4085/1062-605949.2.03

Fernandes, H., Machado, V., Vilaça-Alves, J., Saavedra, F., Aidar, F. y Brustad, R. (2014). Social Support and Sport Injury Recovery: An Overview of Empirical Findings and Practical Implications. Revista de Psicología del Deporte/Journal of Sport Psychology, 23(2), 445-449.

Fuller, C., Ekstrand, J. y Junge, A. (2006). Consensus Statement on Injury Definitions and Data Collection Procedures in Studies of Football (soccer) Injuries. British Journal of Sports Medicine, 40(3), 193-201. http://dx.doi.org/10.1136/bjsm.2006.033282

García-Mas, A., Rubio, V., Fuster-Parra, P., Núñez, A. y Pujals, C. (2014). Determinación de las variables psicológicas y deportivas relevantes a las lesiones deportivas [Determination of the Psychological and Sportive Variables Related to sports Injuries: A Bayesian Analysis] Revista de Psicología del Deporte/Journal of Sport Psychology, 23, 423-429.

Hardy, C., Richman, J. y Rosenfeld, L. (1991). The Role of Social Support in the Life Stress/Injury Relationship. The Sport Psychologist, 5(2), 128-139. http://dx.doi.org/10.1123/tsp.5.2.128

Hurtubise, J. M., Beech, C. y Macpherson, A. (2015). Comparing Severe Injuries by Sex and Sport in Collegiate-Level Athletes: A Descriptive Epidemiologic Study. International Journal of Athletic Therapy \& Training, 20(4), 44-50. http://dx.doi.org/10.1123/ijatt.2014-0090.

Johnson, U. (2007). Psychosocial Antecedents of Sport Injury, Prevention, and Intervention: An Overview 
of Theoretical Approaches and Empirical Findings. International Journal of Sport and Exercise Psychology, 5(4), 352-369. http://dx.doi.org/10.1080/1612197X.2007.9671841

Johnson, U. e Ivarsson, A. (2011). Psychological Predictors of Sport Injuries among Junior Soccer Players. Scandinavian Journal of Medicine \& Science in Sports, 21(1), 129-136. http://dx.doi.org/10.1111/j.1600-0838.2009.01057.x

Johnson, U., Tranaeus, U. e Ivarsson, A. (2014). Current Status and Future Challenges in Psychological Research of Sport Injury Prediction and Prevention: A methodological Perspective. Revista de Psicología del Deporte/Journal of Sport Psychology, 23(2), 401-409.

Junge, A., Engebretsen, L., Mountjoy, M., Alonso, J., Renstrom, P., Aubry, M. y Dvorak, J. (2009). Sports Injuries During the Summer Olympic Games 2008. The American Journal of Sports Medicine, $37(11)$, 2165-2172. http://dx.doi.org/10.1177/0363546509339357

Landeta, O. y Calvete, E. (2002). Adaptación y Validación de la Escala Multidimensional de Apoyo Social Percibido [Adaptation and Validation of the Multidimensional Scale of Perceived Social Support]. Ansiedad y Estrés, 8(2-3), 30-41.

Maddison, R. y Prapavessis, H. (2005). A Psychological Approach to the Prediction and Prevention of Athletic Injury. Journal of Sport and Exercise Psychology, 27(3), 289-310. http://dx.doi.org/10.1123/jsep.27.3.289

Malinauskas, R. (2010). The Associations Among Social Support, Stress, and Life Satisfaction as Perceived by Injured College Athletes. Social Behavior and Personality: An International Journal, 38(6), 741752. http://dx.doi.org/10.2224/sbp.2010.38.6.741

Meeuwisse, W. H., Tyreman, H., Hagel, B. y Emery, C. (2007). A Dynamic Model of Etiology in Sport Injury: The Recursive Nature of Risk and
Causation. Clinical Journal of Sports Medicine, 17(3), 215-219. http://dx.doi.org/10.1097/JSM.0b013e3180592a48

Olmedilla, A. (2005). Factores psicológicos y lesiones en futbolistas: un estudio correlacional [Psychological Factors and Injuries in Soccer Players: A Correlational Study] ( $1^{\text {a }}$ Ed.). Murcia, España: Quaderna.

Olmedilla, A. y García-Mas, A. (2009). El Modelo Global Psicológico de las Lesiones Deportivas [A Global Psychological Model of the Sportive Injuries]. Acción Psicológica, 6(2), 77-91. http://dx.doi.org/10.5944/ap.6.2.223

Olmedilla, A., García-Montalvo, C. y Martínez-Sánchez, F. (2006). Factores psicológicos y vulnerabilidad a las lesiones deportivas: un estudio en futbolistas [Psychological Factors and Vulnerability to Sports Injuries in Soccer Player]. Revista de Psicología del Deporte/Journal of Sport Psychology, 15(1), 37-52.

Olmedilla, A., Ortega, E. y Gómez, J. M. (2014). Influencia de la lesión deportiva en los cambios del estado de ánimo y de la ansiedad precompetitiva en futbolistas [Influence of Sports Injury Changes in Mood and Precompetitive Anxiety in Soccer Players]. Cuadernos de Psicología del Deporte, 14(1), 55-62.

Olmedilla, A., Rubio, V. J., Ortega, E. y García-Mas, A. (2017). Effectiveness of a Stress Management Pilot Program Aimed at Reducing the Incidence of Sports Injuries in Young Football (Soccer) Players. Physical Therapy in Sport, 24, 53-59. http://dx.doi.org/10.1016/j.ptsp.2016.09.003.

Padegimas, E. M., Stepan, J. G., Stoker, G. E., Polites, G. M. y Brophy, R. H. (2016). Epidemiology and Severity of Sports and Recreation Injuries Presenting to a Tertiary Adult Emergency Department. The Physician and Sports Medicine, 44(3), 263-268. http://dx.doi.org/10.1080/00913847.2016.1171683. 
Petrie, T. y Perna, F. (2004). Psychology of injury: Theory, Research, and Practice. En T. Morris y J. Summers, Sport psychology: Theory, Applications, and Issues (pp. 547-571). Australia: Wiley.

Petrie, T., Deiters, J. y Harmison, R. (2014). Mental Toughness, Social Support, and Athletic Identity: Moderators of the Life Stress-Injury Relationship in Collegiate Football Players. Sport Exercise, and Performance Psychology, 3(1), 13-27. http://dx.doi.org/10.1037/a0032698

Pujals, C., Rubio, V. J., Marquez, M. O., SánchezIglesias, I. y Ruiz, R. (2016). Comparative Sport Injury Epidemiological Study on a Spanish Sample of 25 Different Sports. Revista de Psicología del Deporte/Journal of Sport Psychology, 25(2), 271279.

Rees, T., Mitchel, I., Evans, L. y Hardy, L. (2010). Stressors, Social Support and Psychological Responses to Sport Injury in High- and LowPerformance Standard Participants. Psychology of Sport and Exercise, 11(6), 505-512. http://dx.doi.org/10.1016/j.psychsport.2010.07.002

Robbins, J. E. y Rosenfeld, L. B. (2001). Athletes' Perceptions of Social Support Provided by Their Head Coach, Assistant Coach, and Athletic Trainer, Pre-Injury and During Rehabilitation. Journal of Sport Behavior, 24(3), 112-119.

Sheu, Y., Chen, L. H. y Hedegaard, H. (2016). Sports and Recreation Related Injury Episodes in the United States, 2011-2014. National Health Statistics Reports, 99, 1-12.

Udry, E. y Andersen, M. B. (2008). Athletic injury and sport behavior. En T. S. Horn, Advances in Sport Psychology (pp. 401-422). Champaign, Il: Human Kinetics.

Wiese-Bjornstal, D. M., Smith, A. M., Shaffer, S. M. y Morrey, M. A. (1998). An Integrated Model of Response to Sport Injury: Psychological and Sociological Dynamics. Journal of Applied Sport
Psychology, 10(1), 46-69. http://dx.doi.org/10.1080/10413209808406377

Yang, J., Peek-Asa, C., Lowe, J., Heiden, E. y Foster, D. (2010). Social Support Patterns of Collegiate Athletes before and after Injury. Journal of Athletic Training, 45(4), 372-379. http://dx.doi.org/10.4085/1062-6050-45.4.372

Yang, J., Schaefer, J., Zhang, N., Covassin, T., Ding, K. y Heiden, E. (2014). Social Support from the Athletic Trainer and Symptoms of Depression and Anxiety at Return to Play. Journal of Athletic Training, 49(6), 773-779. http://dx.doi.org/10.4085/1062-605049.3.65

Zimet, G. D., Dahlem, N. W., Zimet, S. G. y Farley, G. K. (1988). The Multidimensional Scale of Perceived Social Support. Journal of Personality Assessment, 52 , 30-41. http://dx.doi.org/10.1207/s15327752jpa5201_2 